\title{
Generalized Hybrid One-Step Block Method Involving Fifth Derivative for Solving Fourth-Order Ordinary Differential Equation Directly
}

\author{
Mohammad Alkasassbeh and Zurni Omar \\ Department of Mathematics, School of Quantitative Sciences, College of Arts and Sciences, Universiti Utara Malaysia, Kedah, Malaysia \\ Correspondence should be addressed to Mohammad Alkasassbeh; mfkasassbeh@yahoo.com
}

Received 23 March 2017; Revised 14 June 2017; Accepted 16 October 2017; Published 12 November 2017

Academic Editor: Mehmet Sezer

Copyright (c) 2017 Mohammad Alkasassbeh and Zurni Omar. This is an open access article distributed under the Creative Commons Attribution License, which permits unrestricted use, distribution, and reproduction in any medium, provided the original work is properly cited.

\begin{abstract}
A general one-step three-hybrid (off-step) points block method is proposed for solving fourth-order initial value problems of ordinary differential equations directly. A power series approximate function is employed for deriving this method. The approximate function is interpolated at $\left\{x_{n}, x_{n+r}, x_{n+s}, x_{n+t}\right\}$ while its fourth and fifth derivatives are collocated at all points $\left\{x_{i}\right\}, i=\{0, r, s, t, 1\}$, in the interval of approximation. Several fourth-order initial value problems of ordinary differential equations are then solved to compare the performance of the proposed method with the derived methods. The analysis of the method reveals that the method is consistent and zero stable concluding that the method is also convergent. The numerical results demonstrate the superiority of the new method over the existing ones in terms of error.
\end{abstract}

\section{Introduction}

In this article, we consider the following general fourth-order initial value problems (IVPs) as shown:

$$
\begin{aligned}
y^{(i v)} & =f\left(x, y, y^{\prime}, y^{\prime \prime}, y^{\prime \prime \prime}\right), \\
y(a) & =\alpha_{1}, \\
y_{0}^{\prime}(a) & =\alpha_{2}, \\
y_{0}^{\prime \prime}(a) & =\alpha_{3}, \\
y_{0}^{\prime \prime \prime}(a) & =\alpha_{4},
\end{aligned}
$$

$$
x \in[a, b],
$$

with the assumption that $f \in C^{5}[a, b]$. Several phenomena in physical fields such as neural networks, electric circuits, and ship dynamics can be expressed in differential equations (DEs) forms (see [1-3]).

Block method is one of the efficient methods proposed in 1953 by [4] to enhance the performance of the numerical methods. In 1967, [5] employed block method to provide the essential starting values needed for the predictor schemes. Subsequently, hybrid methods were initiated by [6], which involve the evaluation of functions off-step (nonstep) points. The introduction of hybrid (off-step) points in block methods has many advantages such as the ability to change step size, utilizing data off-step points, and the most important feature according to [7] is the capability to circumvent zero stability barrier condition ([8]).

To increase the accuracy further and to solve the stiffness problem in ordinary differential equations (ODEs), [9] derived second derivative multistep methods for stiff ODEs. Recently, authors like in $[10,11]$ proposed high derivative methods for the same reason. The former developed block hybrid-second derivative method for stiff systems, while the latter introduced a Simpson's type second derivative method for the solution of a first-order stiff system of IVP. In addition, [12] proposed a continuous fourth derivative method for third-order boundary value problems. Following these scholars' footsteps a new generalized three-hybrid onestep fifth derivative method for solving fourth-order ODEs directly using the approach of interpolation and collocation will be proposed. 
This article consists of five sections: Section 1 is for the introduction, while Section 2 illustrates the method derivation, where we consider three-off-step points and employing the collocation approach. The analysis of the approach is discussed in Section 3 which includes zero stability, order, consistency, and convergence. Section 4 covers the solution of some mathematical problems to show the performance of the developed method. Finally, a brief conclusion is performed in Section 5 .

\section{Development of the Method}

Let the following power series polynomial be the approximate solution of (1)

$$
\psi(x)=\sum_{j=0}^{2 m+l-1} a_{j}\left(\frac{x-x_{n}}{h}\right)^{j},
$$

where $l=4$ and $m=5$, the number of interpolation and collocation points. Now, differentiating (2) four and five times yields

$$
\begin{aligned}
\psi^{i v}(x) & =\sum_{j=4}^{2 m+l-1} \frac{a_{j} j !}{h^{4}(j-4) !}\left(\frac{x-x_{n}}{h}\right)^{j-4} \\
& =f\left(x, y, y^{\prime}, y^{\prime \prime}, y^{\prime \prime \prime}\right), \\
\psi^{v}(x) & =\sum_{j=5}^{2 m+l-1} \frac{a_{j} j !}{h^{5}(j-5) !}\left(\frac{x-x_{n}}{h}\right)^{j-5} \\
& =g\left(x, y, y^{\prime}, y^{\prime \prime}, y^{\prime \prime \prime}\right) .
\end{aligned}
$$

Interpolate (2) at $x_{n+i}=x_{n}+i h, i=\{0, r, s, t\}$, and collocate (3) at all points $x_{n+i}=x_{n}+i h, i=\{0, r, s, t, 1\}$, where $\{r, s, t\} \in$ $(0,1)$ produces a system of equations in matrix form as below

$$
\left[\begin{array}{ccccccccc}
1 & 0 & 0 & 0 & 0 & 0 & 0 & \cdots & 0 \\
1 & r & r^{2} & r^{3} & r^{4} & r^{5} & r^{6} & \cdots & r^{\widehat{v}} \\
1 & s & s^{2} & s^{3} & s^{4} & s^{5} & s^{6} & \cdots & s^{\widehat{v}} \\
1 & t & t^{2} & t^{3} & t^{4} & t^{5} & t^{6} & \cdots & t^{\widehat{v}} \\
0 & 0 & 0 & 0 & \frac{4 !}{0 ! h^{4}} & 0 & 0 & \cdots & 0 \\
0 & 0 & 0 & 0 & \frac{4 !}{0 ! h^{4}} & \frac{5 ! r}{1 ! h^{4}} & \frac{6 ! r^{2}}{2 ! h^{4}} & \cdots & \frac{\widehat{v} ! r^{(\widehat{v}-4)}}{(\widehat{v}-4) ! h^{4}} \\
0 & 0 & 0 & 0 & \frac{4 !}{0 ! h^{4}} & \frac{5 ! s}{1 ! h^{4}} & \frac{6 ! s^{2}}{2 ! h^{4}} & \cdots & \frac{\widehat{v} ! s^{(\hat{v}-4)}}{(\widehat{v}-4) ! h^{4}} \\
0 & 0 & 0 & 0 & \frac{4 !}{0 ! h^{4}} & \frac{5 ! t}{1 ! h^{4}} & \frac{6 ! t^{2}}{2 ! h^{4}} & \cdots & \frac{\widehat{v} ! t^{(\hat{v}-4)}}{(\widehat{v}-4) ! h^{4}} \\
0 & 0 & 0 & 0 & \frac{4 !}{0 ! h^{4}} & \frac{5 !}{1 ! h^{4}} & \frac{6 !}{2 ! h^{4}} & \cdots & \frac{(\widehat{v} !}{(\widehat{v}-4) ! h^{4}} \\
0 & 0 & 0 & 0 & 0 & \frac{5 !}{0 ! h^{5}} & 0 & \cdots & 0 \\
0 & 0 & 0 & 0 & 0 & \frac{5 !}{0 ! h^{5}} & \frac{6 ! r}{1 ! h^{5}} & \cdots & \frac{(\widehat{v}) ! r^{(\hat{v}-5)}}{(\widehat{v}-5) ! h^{5}} \\
0 & 0 & 0 & 0 & 0 & \frac{5 !}{0 ! h^{5}} & \frac{6 ! s}{1 ! h^{5}} & \cdots & \frac{(\widehat{v}) ! s^{(\widehat{v}-5)}}{(\widehat{v}-5) ! h^{5}} \\
0 & 0 & 0 & 0 & 0 & \frac{5 !}{0 ! h^{5}} & \frac{6 ! t}{1 ! h^{5}} & \cdots & \frac{(\widehat{v}) ! t^{(\widehat{v}-5)}}{(\widehat{v}-5) ! h^{5}} \\
0 & 0 & 0 & 0 & 0 & \frac{5 !}{0 ! h^{5}} & \frac{6 !}{1 ! h^{5}} & \cdots & \frac{(\hat{v}) !}{(\widehat{v}-5) ! h^{5}}
\end{array}\right]\left[\begin{array}{c}
a_{0} \\
a_{1} \\
a_{2} \\
a_{3} \\
a_{4} \\
a_{5} \\
a_{6} \\
a_{7} \\
a_{8} \\
a_{9} \\
a_{10} \\
a_{11} \\
a_{12} \\
a_{13}
\end{array}\right]
$$

$$
=\left[\begin{array}{c}
y_{n} \\
y_{n+r} \\
y_{n+s} \\
y_{n+t} \\
f_{n} \\
f_{n+r} \\
f_{n+s} \\
f_{n+t} \\
f_{n+1} \\
g_{n} \\
g_{n+r} \\
g_{n+s} \\
g_{n+t} \\
g_{n+1}
\end{array}\right],
$$

where $\widehat{v}=2 m+l-1$. Matrix manipulation is then employed to solve the resulting system (4) for the unknown coefficients $a_{j}^{\prime} s, j=\{0,1, \ldots, 13\}$. Substituting the obtained values of the coefficients back into (2) yields

$$
\begin{aligned}
\psi(x)= & \sum_{i=0, r, s, t} \alpha_{i} y(x+i h) \\
& +h^{4}\left(\sum_{i=r, s, t} \beta_{i} f(x+i h)+\sum_{i=0}^{1} \beta_{i} f(x+i h)\right) \\
& +h^{5}\left(\sum_{i=r, s, t} \gamma_{i} g(x+i h)+\sum_{i=0}^{1} \gamma_{i} g(x+i h)\right),
\end{aligned}
$$

where $n=0,1,2, \ldots, N-1, h=x_{n}-x_{n-1}$ is the constant step size for the partition $\pi_{N}$ of the interval $[a, b]$ which is given by $\pi_{N}=\left[a=x_{0}<x_{1}<\cdots<x_{N-1}<x_{N}=b\right]$, $\alpha_{i}, \beta_{i}$, and $\gamma_{i}$ are undetermined constants listed in Appendix I in the Supplementary Material (see Supplementary Material available online at https://doi.org/10.1155/2017/7637651). For simplicity we shall use $y(x+i h)=y_{n+i}, f(x+i h)=f_{n+i}$, $g(x+i h)=g_{n+i}$, and $g_{n+i}=f_{n+i}^{\prime}$.

Calculating the first, second, and third derivatives of (5) produces

$$
\begin{aligned}
\psi^{\prime}(x)= & \frac{d}{d x}\left(\sum_{i=0, r, s, t} \alpha_{i} y_{n+i}\right) \\
& +h^{4} \frac{d}{d x}\left(\sum_{i=r, s, t} \beta_{i} f_{n+i}+\sum_{i=0}^{1} \beta_{i} f_{n+i}\right) \\
& +h^{5} \frac{d}{d x}\left(\sum_{i=r, s, t} \gamma_{i} g_{n+i}+\sum_{i=0}^{1} \gamma_{i} g_{n+i}\right),
\end{aligned}
$$




$$
\begin{aligned}
\psi^{\prime \prime}(x)= & \frac{d^{2}}{d x^{2}}\left(\sum_{i=0, r, s, t} \alpha_{i} y_{n+i}\right) \\
& +h^{4} \frac{d^{2}}{d x^{2}}\left(\sum_{i=r, s, t} \beta_{i} f_{n+i}+\sum_{i=0}^{1} \beta_{i} f_{n+i}\right) \\
& +h^{5} \frac{d^{2}}{d x^{2}}\left(\sum_{i=r, s, t} \gamma_{i} g_{n+i}+\sum_{i=0}^{1} \gamma_{i} g_{n+i}\right), \\
\psi^{\prime \prime \prime}(x)= & \frac{d^{3}}{d x^{3}}\left(\sum_{i=0, r, s, t} \alpha_{i} y_{n+i}\right) \\
& +h^{4} \frac{d^{3}}{d x^{3}}\left(\sum_{i=r, s, t} \beta_{i} f_{n+i}+\sum_{i=0}^{1} \beta_{i} f_{n+i}\right) \\
& +h^{5} \frac{d^{3}}{d x^{3}}\left(\sum_{i=r, s, t} \gamma_{i} g_{n+i}+\sum_{i=0}^{1} \gamma_{i} g_{n+i}\right) .
\end{aligned}
$$

Evaluating (5) at the noninterpolating points $\left\{x_{n+t}, x_{n+1}\right\}$ with (6) and (7) at all points $\left\{x_{n+i}\right\}, i=\{0, r, s, t, 1\}$, gives the following general equations in block form:

$$
A^{[0]} Y_{m}^{[1]}=A^{[1]} Y_{m}^{[0]}+\sum_{i=0}^{1} B^{[i]} F_{m}^{[i]}+\sum_{i=0}^{1} D^{[i]} G_{m}^{[i]},
$$

where $A^{[0]}$ is an identity matrix of order 12 and

$$
\begin{aligned}
& A^{[1]} \\
& =\left[\begin{array}{llllllllllllllll}
0 & 0 & 0 & 1 & 0 & 0 & 0 & r h & 0 & 0 & 0 & \frac{r^{2} h^{2}}{2} & 0 & 0 & 0 & \frac{r^{3} h^{3}}{6} \\
0 & 0 & 0 & 1 & 0 & 0 & 0 & s h & 0 & 0 & 0 & \frac{s^{2} h^{2}}{2} & 0 & 0 & 0 & \frac{s^{3} h^{3}}{6} \\
0 & 0 & 0 & 1 & 0 & 0 & 0 & t h & 0 & 0 & 0 & \frac{t^{2} h^{2}}{2} & 0 & 0 & 0 & \frac{t^{3} h^{3}}{6} \\
0 & 0 & 0 & 1 & 0 & 0 & 0 & h & 0 & 0 & 0 & \frac{h^{2}}{2} & 0 & 0 & 0 & \frac{h^{3}}{6} \\
0 & 0 & 0 & 0 & 0 & 0 & 0 & 1 & 0 & 0 & 0 & r h & 0 & 0 & 0 & \frac{r^{2} h^{2}}{2} \\
0 & 0 & 0 & 0 & 0 & 0 & 0 & 1 & 0 & 0 & 0 & s h & 0 & 0 & 0 & \frac{s^{2} h^{2}}{2} \\
0 & 0 & 0 & 0 & 0 & 0 & 0 & 1 & 0 & 0 & 0 & t h & 0 & 0 & 0 & \frac{t^{2} h^{2}}{2} \\
0 & 0 & 0 & 0 & 0 & 0 & 0 & 1 & 0 & 0 & 0 & h & 0 & 0 & 0 & \frac{h^{2}}{2} \\
0 & 0 & 0 & 0 & 0 & 0 & 0 & 0 & 0 & 0 & 0 & 1 & 0 & 0 & 0 & r h \\
0 & 0 & 0 & 0 & 0 & 0 & 0 & 0 & 0 & 0 & 0 & 1 & 0 & 0 & 0 & s h \\
0 & 0 & 0 & 0 & 0 & 0 & 0 & 0 & 0 & 0 & 0 & 1 & 0 & 0 & 0 & t h \\
0 & 0 & 0 & 0 & 0 & 0 & 0 & 0 & 0 & 0 & 0 & 1 & 0 & 0 & 0 & h \\
0 & 0 & 0 & 0 & 0 & 0 & 0 & 0 & 0 & 0 & 0 & 0 & 0 & 0 & 0 & 1 \\
0 & 0 & 0 & 0 & 0 & 0 & 0 & 0 & 0 & 0 & 0 & 0 & 0 & 0 & 0 & 1 \\
0 & 0 & 0 & 0 & 0 & 0 & 0 & 0 & 0 & 0 & 0 & 0 & 0 & 0 & 0 & 1 \\
0 & 0 & 0 & 0 & 0 & 0 & 0 & 0 & 0 & 0 & 0 & 0 & 0 & 0 & 0 & 1
\end{array}\right],
\end{aligned}
$$

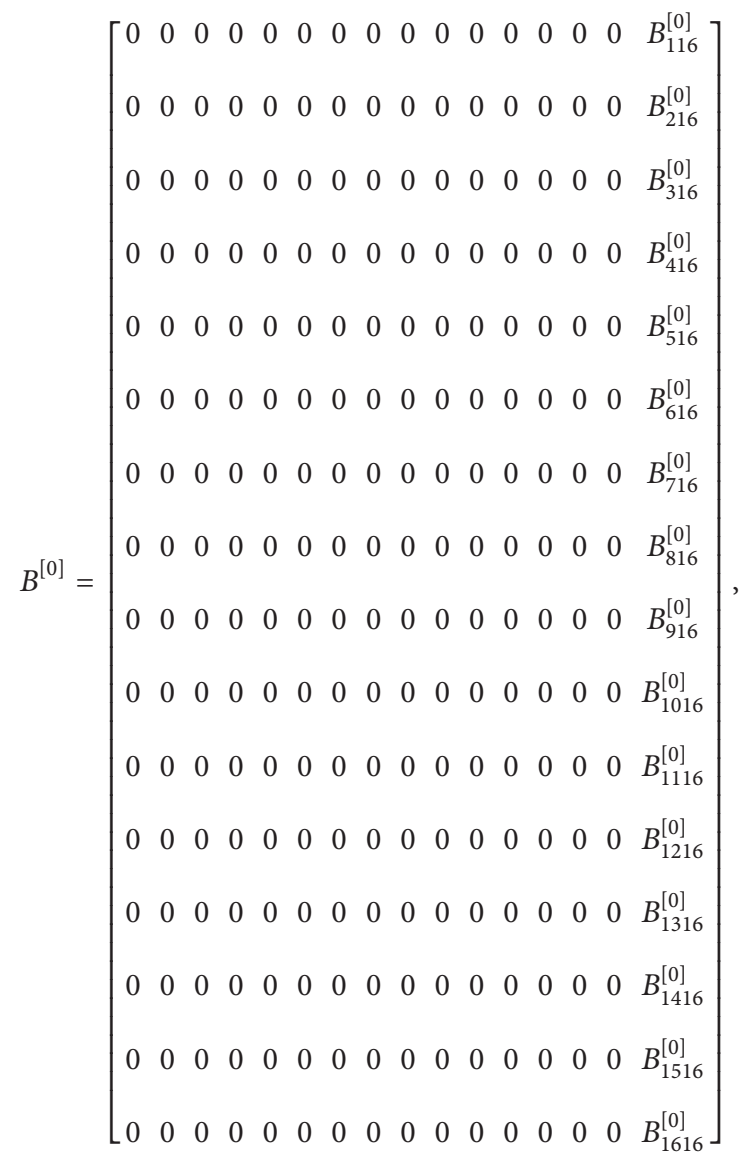

$$
B^{[1]}=\left[\begin{array}{llll}
B_{11}^{[1]} & B_{12}^{[1]} & B_{13}^{[1]} & B_{14}^{[1]} \\
B_{21}^{[1]} & B_{22}^{[1]} & B_{23}^{[1]} & B_{24}^{[1]} \\
B_{31}^{[1]} & B_{32}^{[1]} & B_{33}^{[1]} & B_{34}^{[1]} \\
B_{41}^{[1]} & B_{42}^{[1]} & B_{43}^{[1]} & B_{44}^{[1]} \\
B_{51}^{[1]} & B_{52}^{[1]} & B_{53}^{[1]} & B_{54}^{[1]} \\
B_{61}^{[1]} & B_{62}^{[1]} & B_{63}^{[1]} & B_{64}^{[1]} \\
B_{71}^{[1]} & B_{72}^{[1]} & B_{73}^{[1]} & B_{74}^{[1]} \\
B_{81}^{[1]} & B_{82}^{[1]} & B_{83}^{[1]} & B_{84}^{[1]} \\
B_{91}^{[1]} & B_{92}^{[1]} & B_{93}^{[1]} & B_{94}^{[1]} \\
B_{101}^{[1]} & B_{102}^{[1]} & B_{103}^{[1]} & B_{104}^{[1]} \\
B_{111}^{[1]} & B_{112}^{[1]} & B_{113}^{[1]} & B_{114}^{[1]} \\
B_{121}^{[1]} & B_{122}^{[1]} & B_{123}^{[1]} & B_{124}^{[1]} \\
B_{131}^{[1]} & B_{132}^{[1]} & B_{133}^{[1]} & B_{134}^{[1]} \\
B_{141}^{[1]} & B_{142}^{[1]} & B_{143}^{[1]} & B_{144}^{[1]} \\
B_{151}^{[1]} & B_{152}^{[1]} & B_{153}^{[1]} & B_{154}^{[1]} \\
B_{161}^{[1]} & B_{162}^{[1]} & B_{163}^{[1]} & B_{164}^{[1]}
\end{array}\right],
$$


$D^{[0]}=\left[\begin{array}{llllllllllllllll}0 & 0 & 0 & 0 & 0 & 0 & 0 & 0 & 0 & 0 & 0 & 0 & 0 & 0 & 0 & D_{116}^{[0]} \\ 0 & 0 & 0 & 0 & 0 & 0 & 0 & 0 & 0 & 0 & 0 & 0 & 0 & 0 & 0 & D_{216}^{[0]} \\ 0 & 0 & 0 & 0 & 0 & 0 & 0 & 0 & 0 & 0 & 0 & 0 & 0 & 0 & 0 & D_{316}^{[0]} \\ 0 & 0 & 0 & 0 & 0 & 0 & 0 & 0 & 0 & 0 & 0 & 0 & 0 & 0 & 0 & D_{416}^{[0]} \\ 0 & 0 & 0 & 0 & 0 & 0 & 0 & 0 & 0 & 0 & 0 & 0 & 0 & 0 & 0 & D_{516}^{[0]} \\ 0 & 0 & 0 & 0 & 0 & 0 & 0 & 0 & 0 & 0 & 0 & 0 & 0 & 0 & 0 & D_{616}^{[0]} \\ 0 & 0 & 0 & 0 & 0 & 0 & 0 & 0 & 0 & 0 & 0 & 0 & 0 & 0 & 0 & D_{716}^{[0]} \\ 0 & 0 & 0 & 0 & 0 & 0 & 0 & 0 & 0 & 0 & 0 & 0 & 0 & 0 & 0 & D_{816}^{[0]} \\ 0 & 0 & 0 & 0 & 0 & 0 & 0 & 0 & 0 & 0 & 0 & 0 & 0 & 0 & 0 & D_{916}^{[0]} \\ 0 & 0 & 0 & 0 & 0 & 0 & 0 & 0 & 0 & 0 & 0 & 0 & 0 & 0 & 0 & D_{1016}^{[0]} \\ 0 & 0 & 0 & 0 & 0 & 0 & 0 & 0 & 0 & 0 & 0 & 0 & 0 & 0 & 0 & D_{1116}^{[0]} \\ 0 & 0 & 0 & 0 & 0 & 0 & 0 & 0 & 0 & 0 & 0 & 0 & 0 & 0 & 0 & D_{1216}^{[0]} \\ 0 & 0 & 0 & 0 & 0 & 0 & 0 & 0 & 0 & 0 & 0 & 0 & 0 & 0 & 0 & D_{1316}^{[0]} \\ 0 & 0 & 0 & 0 & 0 & 0 & 0 & 0 & 0 & 0 & 0 & 0 & 0 & 0 & 0 & D_{1416}^{[0]} \\ 0 & 0 & 0 & 0 & 0 & 0 & 0 & 0 & 0 & 0 & 0 & 0 & 0 & 0 & 0 & D_{1516}^{[0]} \\ 0 & 0 & 0 & 0 & 0 & 0 & 0 & 0 & 0 & 0 & 0 & 0 & 0 & 0 & 0 & D_{1616}^{[0]}\end{array}\right]$,

$D^{[1]}=\left[\begin{array}{cccc}D_{11}^{[1]} & D_{12}^{[1]} & D_{13}^{[1]} & D_{14}^{[1]} \\ D_{21}^{[1]} & D_{22}^{[1]} & D_{23}^{[1]} & D_{24}^{[1]} \\ D_{31}^{[1]} & D_{32}^{[1]} & D_{33}^{[1]} & D_{34}^{[1]} \\ D_{41}^{[1]} & D_{42}^{[1]} & D_{43}^{[1]} & D_{44}^{[1]} \\ D_{51}^{[1]} & D_{52}^{[1]} & D_{53}^{[1]} & D_{54}^{[1]} \\ D_{61}^{[1]} & D_{62}^{[1]} & D_{63}^{[1]} & D_{64}^{[1]} \\ D_{71}^{[1]} & D_{72}^{[1]} & D_{73}^{[1]} & D_{74}^{[1]} \\ D_{81}^{[1]} & D_{82}^{[1]} & D_{83}^{[1]} & D_{84}^{[1]} \\ D_{91}^{[1]} & D_{92}^{[1]} & D_{93}^{[1]} & D_{94}^{[1]} \\ D_{101}^{[1]} & D_{102}^{[1]} & D_{103}^{[1]} & D_{104}^{[1]} \\ D_{111}^{[1]} & D_{112}^{[1]} & D_{113}^{[1]} & D_{114}^{[1]} \\ D_{121}^{[1]} & D_{122}^{[1]} & D_{123}^{[1]} & D_{124}^{[1]} \\ D_{131}^{[1]} & D_{132}^{[1]} & D_{133}^{[1]} & D_{134}^{[1]} \\ D_{141}^{[1]} & D_{142}^{[1]} & D_{143}^{[1]} & D_{144}^{[1]} \\ D_{151}^{[1]} & D_{152}^{[1]} & D_{153}^{[1]} & D_{154}^{[1]} \\ D_{161}^{[1]} & D_{162}^{[1]} & D_{163}^{[1]} & D_{164}^{[1]}\end{array}\right]$.
The entries of $B^{[0]}, B^{[1]}, D^{[0]}$, and $D^{[1]}$ are listed in Appendix II in the Supplementary Material section, while the vectors $Y_{m}^{[0]}, Y_{m}^{[1]}, F_{m}^{[0]}, F_{m}^{[1]}, G_{m}^{[0]}, G_{m}^{[1]}$ are defined as follows:
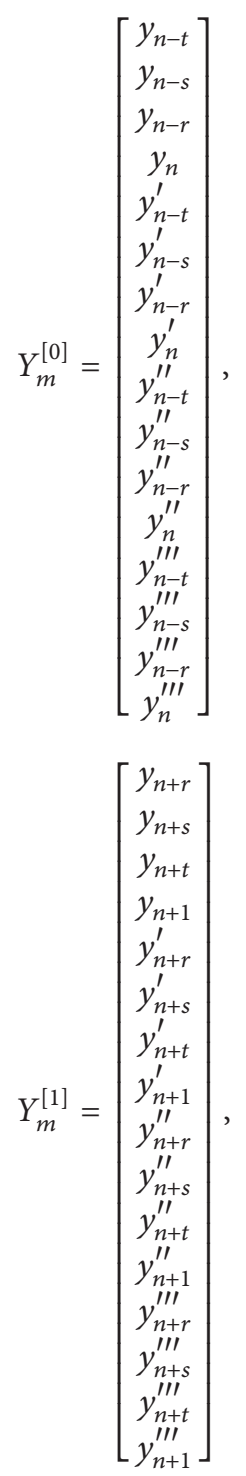

$F_{m}^{[0]}=\left[\begin{array}{c}f_{n-15} \\ f_{n-14} \\ f_{n-13} \\ f_{n-12} \\ f_{n-11} \\ f_{n-10} \\ f_{n-9} \\ f_{n-8} \\ f_{n-7} \\ f_{n-6} \\ f_{n-5} \\ f_{n-4} \\ f_{n-3} \\ f_{n-2} \\ f_{n-1} \\ f_{n}\end{array}\right]$, 


$$
\begin{aligned}
F_{m}^{[1]} & =\left[\begin{array}{c}
f_{n+r} \\
f_{n+s} \\
f_{n+t} \\
f_{n+1}
\end{array}\right], \\
G_{m}^{[0]} & =\left[\begin{array}{c}
g_{n-15} \\
g_{n-14} \\
g_{n-13} \\
g_{n-12} \\
g_{n-11} \\
g_{n-10} \\
g_{n-9} \\
g_{n-8} \\
g_{n-7} \\
g_{n-6} \\
g_{n-5} \\
g_{n-4} \\
g_{n-3} \\
g_{n-2} \\
g_{n-1} \\
g_{n}
\end{array}\right],
\end{aligned}
$$

$$
G_{m}^{[1]}=\left[\begin{array}{c}
g_{n+r} \\
g_{n+s} \\
g_{n+t} \\
g_{n+1}
\end{array}\right] .
$$

\section{Analysis of the Method}

\subsection{Zero Stability}

Definition 1. The hybrid block method formula (9) is said to be zero stable if no root $R_{m}$ of the first characteristic equation $\rho(R)$ has modulus greater than one; that is, $\left|R_{m}\right| \leqslant 1$, and if $R_{m}=1$ then the multiplicity of $R_{m}$ must not exceed four.

To prove that the roots of the first characteristic equation satisfy the previous definition

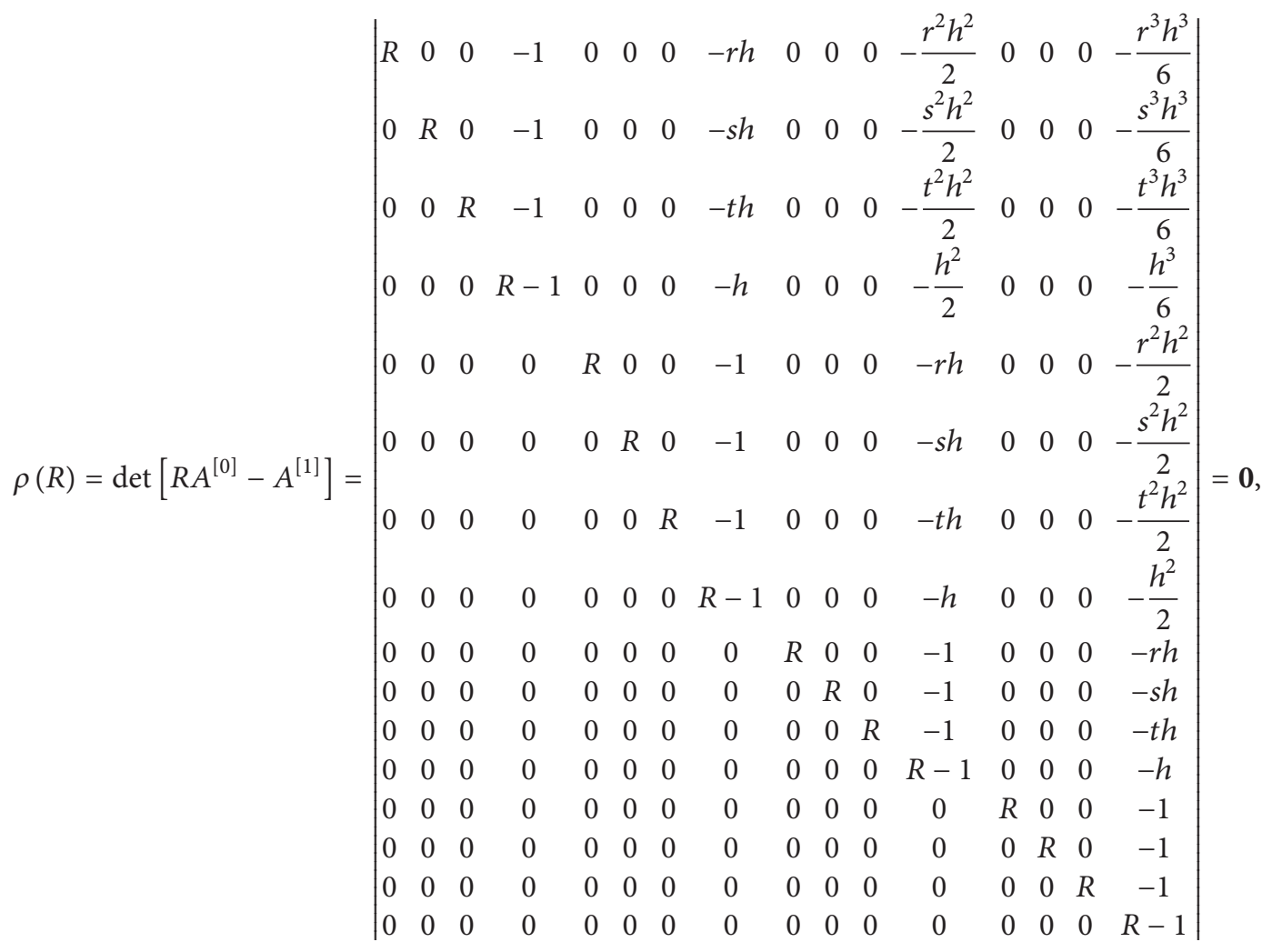

which imply that

$$
\begin{aligned}
R^{12}(R-1)^{4} & =0 \Longrightarrow \\
R_{m} & = \begin{cases}0, & \text { if } m=1(1) 12, \\
1, & \text { if } m=13(1) 16 .\end{cases}
\end{aligned}
$$

As a result, the developed method is zero stable.
3.2. Order of the Method. The linear operator $\Theta$ associated with the hybrid block methods formula (9) is defined as

$$
\begin{aligned}
\Theta\{y(x) ; h\}= & A^{[0]} Y_{m}^{[1]}-A^{[1]} Y_{m}^{[0]}-\sum_{i=0}^{1} B^{[i]} F_{m}^{[i]} \\
& -\sum_{i=0}^{1} D^{[i]} G_{m}^{[i]} .
\end{aligned}
$$


Expanding the above equation in Taylor series and combining like terms imply

$$
\begin{aligned}
\Theta\{y(x) ; h\}= & \widehat{C}_{0} h^{0} y(x)+\widehat{C}_{1} h^{1} y^{\prime}(x)+\widehat{C}_{2} h^{2} y^{\prime \prime}(x) \\
& +\cdots+\widehat{C}_{p+4} h^{p+4} y^{(p+4)}(x)+\cdots .
\end{aligned}
$$

According to $[7,13]$ method (9) is said to be of order $p$ if

$$
\widehat{C}_{0}=\widehat{C}_{1}=\cdots=\widehat{C}_{p+3}=0
$$

$$
\widehat{C}_{p+4} \neq 0
$$

The term $\widehat{C}_{p+4}$ is called the error constant and the local truncation error is given by

$$
t_{n+k}=\widehat{C}_{p+4} y^{(p+4)} h^{p+4}+O\left(h^{p+5}\right) .
$$

Comparing like terms of $y^{(i)}$ and $h^{i}$ in (15) produces the coefficients $\widehat{C}_{0}=\widehat{C}_{1}=\cdots=\widehat{C}_{13}=0$ with vector of error constants

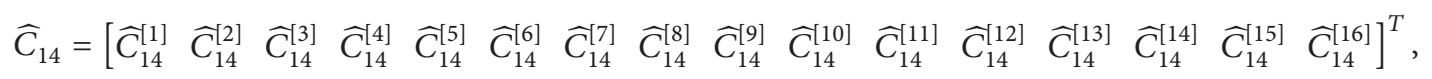

where

$$
\begin{aligned}
& \widehat{C}_{14}^{[1]}=\frac{r^{8}}{7846046208000}\left(20 r^{6}-70 r^{5} s-70 r^{5} t-70 r^{5}\right. \\
& +65 r^{4} s^{2}+260 r^{4} s t+260 r^{4} s+65 r^{4} t^{2}+260 r^{4} t \\
& +65 r^{4}-260 r^{3} s^{2} t-260 r^{3} s^{2}-260 r^{3} s t^{2} \\
& -1040 r^{3} s t-260 r^{3} s-260 r^{3} t^{2}-260 r^{3} t \\
& +286 r^{2} s^{2} t^{2}+1144 r^{2} s^{2} t+286 r^{2} s^{2}+1144 r^{2} s t^{2} \\
& +1144 r^{2} s t+286 r^{2} t^{2}-1430 r s^{2} t^{2}-1430 r s^{2} t \\
& \left.-1430 r s t^{2}+2145 s^{2} t^{2}\right) \text {, } \\
& \widehat{C}_{14}^{[2]}=\frac{s^{8}}{7846046208000}\left(65 r^{2} s^{4}-260 r^{2} s^{3} t-260 r^{2} s^{3}\right. \\
& +286 r^{2} s^{2} t^{2}+1144 r^{2} s^{2} t+286 r^{2} s^{2}-1430 r^{2} s t^{2} \\
& -1430 r^{2} s t+2145 r^{2} t^{2}-70 r s^{5}+260 r s^{4} t+260 r s^{4} \\
& -260 r s^{3} t^{2}-1040 r s^{3} t-260 r s^{3}+1144 r s^{2} t^{2} \\
& +1144 r s^{2} t-1430 r s t^{2}+20 s^{6}-70 s^{5} t-70 s^{5} \\
& +65 s^{4} t^{2}+260 s^{4} t+65 s^{4}-260 s^{3} t^{2}-260 s^{3} t \\
& \left.+286 s^{2} t^{2}\right) \\
& \widehat{C}_{14}^{[3]}=\frac{t^{8}}{7846046208000}\left(286 r^{2} s^{2} t^{2}-1430 r^{2} s^{2} t\right. \\
& +2145 r^{2} s^{2}-260 r^{2} s t^{3}+1144 r^{2} s t^{2}-1430 r^{2} s t \\
& +65 r^{2} t^{4}-260 r^{2} t^{3}+286 r^{2} t^{2}-260 r s^{2} t^{3} \\
& +1144 r s^{2} t^{2}-1430 r s^{2} t+260 r s t^{4}-1040 r s t^{3} \\
& +1144 r s t^{2}-70 r t^{5}+260 r t^{4}-260 r t^{3}+65 s^{2} t^{4} \\
& -260 s^{2} t^{3}+286 s^{2} t^{2}-70 s t^{5}+260 s t^{4}-260 s t^{3} \\
& \left.+20 t^{6}-70 t^{5}+65 t^{4}\right)
\end{aligned}
$$

$$
\begin{aligned}
\widehat{C}_{14}^{[4]} & =\frac{1}{7846046208000}\left(2145 r^{2} s^{2} t^{2}-1430 r^{2} s^{2} t\right. \\
& +286 r^{2} s^{2}-1430 r^{2} s t^{2}+1144 r^{2} s t-260 r^{2} s \\
& +286 r^{2} t^{2}-260 r^{2} t+65 r^{2}-1430 r s^{2} t^{2}+1144 r s^{2} t \\
& -260 r s^{2}+1144 r s t^{2}-1040 r s t+260 r s-260 r t^{2} \\
& +260 r t-70 r+286 s^{2} t^{2}-260 s^{2} t+65 s^{2}-260 s t^{2} \\
& \left.+260 s t-70 s+65 t^{2}-70 t+20\right) \\
\widehat{C}_{14}^{5]} & =\frac{r^{7}}{1307674368000}\left(28 r^{6}-91 r^{5} s-91 r^{5} t-91 r^{5}\right. \\
& +78 r^{4} s^{2}+312 r^{4} s t+312 r^{4} s+78 r^{4} t^{2}+312 r^{4} t \\
& +78 r^{4}-286 r^{3} s^{2} t-286 r^{3} s^{2}-286 r^{3} s t^{2} \\
& -1144 r^{3} s t-286 r^{3} s-286 r^{3} t^{2}-286 r^{3} t \\
& +286 r^{2} s^{2} t^{2}+1144 r^{2} s^{2} t+286 r^{2} s^{2}+1144 r^{2} s t^{2} \\
& +1144 r^{2} s t+286 r^{2} t^{2}-1287 r s^{2} t^{2}-1287 r s^{2} t
\end{aligned}
$$$$
\widehat{C}_{14}^{[6]}=\frac{s^{7}}{1307674368000}\left(78 r^{2} s^{4}-286 r^{2} s^{3} t-286 r^{2} s^{3}\right.
$$$$
+286 r^{2} s^{2} t^{2}+1144 r^{2} s^{2} t+286 r^{2} s^{2}-1287 r^{2} s t^{2}
$$$$
-1287 r^{2} s t+1716 r^{2} t^{2}-91 r s^{5}+312 r s^{4} t+312 r s^{4}
$$$$
-286 r s^{3} t^{2}-1144 r s^{3} t-286 r s^{3}+1144 r s^{2} t^{2}
$$$$
+1144 r s^{2} t-1287 r s t^{2}+28 s^{6}-91 s^{5} t-91 s^{5}
$$$$
+78 s^{4} t^{2}+312 s^{4} t+78 s^{4}-286 s^{3} t^{2}-286 s^{3} t
$$$$
\left.+286 s^{2} t^{2}\right)
$$ 


$$
\begin{aligned}
& \widehat{C}_{14}^{[7]}=\frac{t^{7}}{1307674368000}\left(286 r^{2} s^{2} t^{2}-1287 r^{2} s^{2} t\right. \\
& +1716 r^{2} s^{2}-286 r^{2} s t^{3}+1144 r^{2} s t^{2}-1287 r^{2} s t \\
& +78 r^{2} t^{4}-286 r^{2} t^{3}+286 r^{2} t^{2}-286 r s^{2} t^{3} \\
& +1144 r s^{2} t^{2}-1287 r s^{2} t+312 r s t^{4}-1144 r s t^{3} \\
& +1144 r s t^{2}-91 r t^{5}+312 r t^{4}-286 r t^{3}+78 s^{2} t^{4} \\
& -286 s^{2} t^{3}+286 s^{2} t^{2}-91 s t^{5}+312 s t^{4}-286 s t^{3} \\
& \left.+28 t^{6}-91 t^{5}+78 t^{4}\right) \text {, } \\
& \widehat{C}_{14}^{[8]}=\frac{1}{1307674368000}\left(1716 r^{2} s^{2} t^{2}-1287 r^{2} s^{2} t\right. \\
& +286 r^{2} s^{2}-1287 r^{2} s t^{2}+1144 r^{2} s t-286 r^{2} s \\
& +286 r^{2} t^{2}-286 r^{2} t+78 r^{2}-1287 r s^{2} t^{2}+1144 r s^{2} t \\
& -286 r s^{2}+1144 r s t^{2}-1144 r s t+312 r s-286 r t^{2} \\
& +312 r t-91 r+286 s^{2} t^{2}-286 s^{2} t+78 s^{2}-286 s t^{2} \\
& \left.+312 s t-91 s+78 t^{2}-91 t+28\right), \\
& \widehat{C}_{14}^{[9]}=\frac{r^{6}}{100590336000}\left(14 r^{6}-42 r^{5} s-42 r^{5} t-42 r^{5}\right. \\
& +33 r^{4} s^{2}+132 r^{4} s t+132 r^{4} s+33 r^{4} t^{2}+132 r^{4} t \\
& +33 r^{4}-110 r^{3} s^{2} t-110 r^{3} s^{2}-110 r^{3} s t^{2}-440 r^{3} s t \\
& -110 r^{3} s-110 r^{3} t^{2}-110 r^{3} t+99 r^{2} s^{2} t^{2}+396 r^{2} s^{2} t \\
& +99 r^{2} s^{2}+396 r^{2} s t^{2}+396 r^{2} s t+99 r^{2} t^{2}-396 r s^{2} t^{2} \\
& \left.-396 r s^{2} t-396 r s t^{2}+462 s^{2} t^{2}\right) \text {, } \\
& \widehat{C}_{14}^{[10]}=\frac{s^{6}}{100590336000}\left(33 r^{2} s^{4}-110 r^{2} s^{3} t-110 r^{2} s^{3}\right. \\
& +99 r^{2} s^{2} t^{2}+396 r^{2} s^{2} t+99 r^{2} s^{2}-396 r^{2} s t^{2} \\
& -396 r^{2} s t+462 r^{2} t^{2}-42 r s^{5}+132 r s^{4} t+132 r s^{4} \\
& -110 r s^{3} t^{2}-440 r s^{3} t-110 r s^{3}+396 r s^{2} t^{2} \\
& +396 r s^{2} t-396 r s t^{2}+14 s^{6}-42 s^{5} t-42 s^{5} \\
& +33 s^{4} t^{2}+132 s^{4} t+33 s^{4}-110 s^{3} t^{2}-110 s^{3} t \\
& \left.+99 s^{2} t^{2}\right) \text {, } \\
& \widehat{C}_{14}^{[11]}=\frac{t^{6}}{100590336000}\left(99 r^{2} s^{2} t^{2}-396 r^{2} s^{2} t\right. \\
& +462 r^{2} s^{2}-110 r^{2} s t^{3}+396 r^{2} s t^{2}-396 r^{2} s t \\
& +33 r^{2} t^{4}-110 r^{2} t^{3}+99 r^{2} t^{2}-110 r s^{2} t^{3}+396 r s^{2} t^{2} \\
& \begin{aligned}
& -396 r s^{2} t+132 r s t^{4}-440 r s t^{3}+396 r s t^{2}-42 r t^{5} \\
+ & 132 r t^{4}-110 r t^{3}+33 s^{2} t^{4}-110 s^{2} t^{3}+99 s^{2} t^{2} \\
& \left.-42 s t^{5}+132 s t^{4}-110 s t^{3}+14 t^{6}-42 t^{5}+33 t^{4}\right), \\
\widehat{C}_{14}^{112]}= & \frac{1}{100590336000}\left(462 r^{2} s^{2} t^{2}-396 r^{2} s^{2} t\right. \\
+ & 99 r^{2} s^{2}-396 r^{2} s t^{2}+396 r^{2} s t-110 r^{2} s+99 r^{2} t^{2} \\
& -110 r^{2} t+33 r^{2}-396 r s^{2} t^{2}+396 r s^{2} t-110 r s^{2} \\
+ & 396 r s t^{2}-440 r s t+132 r s-110 r t^{2}+132 r t \\
- & 42 r+99 s^{2} t^{2}-110 s^{2} t+33 s^{2}-110 s t^{2}+132 s t \\
- & \left.42 s+33 t^{2}-42 t+14\right), \\
\widehat{C}_{14}^{13]} & =\frac{r^{5}}{50295168000}\left(28 r^{6}-77 r^{5} s-77 r^{5} t-77 r^{5}\right. \\
+ & 55 r^{4} s^{2}+220 r^{4} s t+220 r^{4} s+55 r^{4} t^{2}+220 r^{4} t \\
+ & 55 r^{4}-165 r^{3} s^{2} t-165 r^{3} s^{2}-165 r^{3} s t^{2}-660 r^{3} s t \\
- & 165 r^{3} s-165 r^{3} t^{2}-165 r^{3} t+132 r^{2} s^{2} t^{2} \\
+ & 528 r^{2} s^{2} t+132 r^{2} s^{2}+528 r^{2} s t^{2}+528 r^{2} s t \\
+ & 132 r^{2} t^{2}-462 r s^{2} t^{2}-462 r s^{2} t-462 r s t^{2} \\
+ & \left.462 s^{2} t^{2}\right),
\end{aligned} \\
& \widehat{C}_{14}^{[14]}=\frac{s^{5}}{50295168000}\left(55 r^{2} s^{4}-165 r^{2} s^{3} t-165 r^{2} s^{3}\right. \\
& +132 r^{2} s^{2} t^{2}+528 r^{2} s^{2} t+132 r^{2} s^{2}-462 r^{2} s t^{2} \\
& -462 r^{2} s t+462 r^{2} t^{2}-77 r s^{5}+220 r s^{4} t+220 r s^{4} \\
& -165 r s^{3} t^{2}-660 r s^{3} t-165 r s^{3}+528 r s^{2} t^{2} \\
& +528 r s^{2} t-462 r s t^{2}+28 s^{6}-77 s^{5} t-77 s^{5} \\
& +55 s^{4} t^{2}+220 s^{4} t+55 s^{4}-165 s^{3} t^{2}-165 s^{3} t \\
& \left.+132 s^{2} t^{2}\right) \text {, } \\
& \widehat{C}_{14}^{[15]}=\frac{t^{5}}{50295168000}\left(132 r^{2} s^{2} t^{2}-462 r^{2} s^{2} t\right. \\
& +462 r^{2} s^{2}-165 r^{2} s t^{3}+528 r^{2} s t^{2}-462 r^{2} s t \\
& +55 r^{2} t^{4}-165 r^{2} t^{3}+132 r^{2} t^{2}-165 r s^{2} t^{3} \\
& +528 r s^{2} t^{2}-462 r s^{2} t+220 r s t^{4}-660 r s t^{3} \\
& +528 r s t^{2}-77 r t^{5}+220 r t^{4}-165 r t^{3}+55 s^{2} t^{4} \\
& -165 s^{2} t^{3}+132 s^{2} t^{2}-77 s t^{5}+220 s t^{4}-165 s t^{3} \\
& \left.+28 t^{6}-77 t^{5}+55 t^{4}\right) \text {, }
\end{aligned}
$$




$$
\begin{aligned}
& \widehat{C}_{14}^{[16]}=\frac{1}{50295168000}\left(462 r^{2} s^{2} t^{2}-462 r^{2} s^{2} t\right. \\
& \quad+132 r^{2} s^{2}-462 r^{2} s t^{2}+528 r^{2} s t-165 r^{2} s \\
& \quad+132 r^{2} t^{2}-165 r^{2} t+55 r^{2}-462 r s^{2} t^{2}+528 r s^{2} t \\
& \quad-165 r s^{2}+528 r s t^{2}-660 r s t+220 r s-165 r t^{2} \\
& +220 r t-77 r+132 s^{2} t^{2}-165 s^{2} t+55 s^{2}-165 s t^{2} \\
& \left.+220 s t-77 s+55 t^{2}-77 t+28\right),
\end{aligned}
$$

which concludes that the order of the developed method is $p=10$.

\subsection{Consistency}

Definition 2. A block method is said to be consistent if its order $p$ is greater than one.

Consistency property is achieved for the hybrid block method from the above analysis since the order $p=10 \geq 1$.

\subsection{Convergence}

Theorem 3 (see [14]). Consistency and zero stability are sufficient conditions for a linear multistep method to be convergent.

The hybrid block method (9) is convergent since it fulfils both the consistency and zero stability conditions.

3.5. Region of Absolute Stability. Stability region of the hybrid block (9) is discussed in spirit of [7]. The test problem of the forms $y^{\prime}=\lambda y, y^{\prime \prime}=\lambda^{2} y, y^{\prime \prime \prime}=\lambda^{3} y, y^{i v}=\lambda^{4} y$, and $y^{v}=$ $\lambda^{5} y$, where $\lambda<0$ are substituted into the main methods of block (9), is shown below

$$
\bar{A}^{[0]} \bar{Y}_{m+1}=\sum_{i=1}^{4} \bar{A}^{[i]} \bar{Y}_{m}^{[i]}+\sum_{i=0}^{1} \bar{B}^{[i]} \bar{F}_{m}^{[i]}+\sum_{i=0}^{1} \bar{D}^{[i]} \bar{G}_{m}^{[i]},
$$

where

$$
\begin{aligned}
& \bar{A}^{[0]}=\left[\begin{array}{llll}
1 & 0 & 0 & 0 \\
0 & 1 & 0 & 0 \\
0 & 0 & 1 & 0 \\
0 & 0 & 0 & 1
\end{array}\right], \\
& \bar{A}^{[1]}=\left[\begin{array}{llll}
0 & 0 & 0 & 1 \\
0 & 0 & 0 & 1 \\
0 & 0 & 0 & 1 \\
0 & 0 & 0 & 1
\end{array}\right], \\
& \bar{A}^{[2]}=\left[\begin{array}{llll}
0 & 0 & 0 & r \\
0 & 0 & 0 & s \\
0 & 0 & 0 & t \\
0 & 0 & 0 & 1
\end{array}\right],
\end{aligned}
$$

$$
\begin{aligned}
\bar{A}^{[3]} & =\left[\begin{array}{llll}
0 & 0 & 0 & \frac{r^{2}}{2} \\
0 & 0 & 0 & \frac{s^{2}}{2} \\
0 & 0 & 0 & \frac{t^{2}}{2} \\
0 & 0 & 0 & \frac{1}{2}
\end{array}\right], \\
\bar{A}^{[4]} & =\left[\begin{array}{llll}
0 & 0 & 0 & \frac{r^{3}}{6} \\
0 & 0 & 0 & \frac{s^{3}}{6} \\
0 & 0 & 0 & \frac{t^{3}}{6} \\
0 & 0 & 0 & \frac{1}{6}
\end{array}\right],
\end{aligned}
$$

$$
\bar{B}^{[0]}=\frac{1}{h^{4}}\left[\begin{array}{cccc}
0 & 0 & 0 & B_{116}^{[0]} \\
0 & 0 & 0 & B_{216}^{[0]} \\
0 & 0 & 0 & B_{316}^{[0]} \\
0 & 0 & 0 & B_{416}^{[0]}
\end{array}\right] \text {, }
$$

$$
\bar{B}^{[1]}=\frac{1}{h^{4}}\left[\begin{array}{cccc}
B_{11}^{[1]} & B_{12}^{[1]} & B_{13}^{[1]} & B_{14}^{[1]} \\
B_{21}^{[1]} & B_{22}^{[1]} & B_{23}^{[1]} & B_{24}^{[1]} \\
B_{31}^{[1]} & B_{32}^{[1]} & B_{33}^{[1]} & B_{34}^{[1]} \\
B_{41}^{[1]} & B_{42}^{[1]} & B_{43}^{[1]} & B_{44}^{[1]}
\end{array}\right] \text {, }
$$

$$
\bar{D}^{[0]}=\frac{1}{h^{5}}\left[\begin{array}{cccc}
0 & 0 & 0 & D_{116}^{[0]} \\
0 & 0 & 0 & D_{216}^{[0]} \\
0 & 0 & 0 & D_{316}^{[0]} \\
0 & 0 & 0 & D_{416}^{[0]}
\end{array}\right] \text {, }
$$

$$
\bar{D}^{[1]}=\frac{1}{h^{5}}\left[\begin{array}{cccc}
D_{11}^{[1]} & D_{12}^{[1]} & D_{13}^{[1]} & D_{14}^{[1]} \\
D_{21}^{[1]} & D_{22}^{[1]} & D_{23}^{[1]} & D_{24}^{[1]} \\
D_{31}^{[1]} & D_{32}^{[1]} & D_{33}^{[1]} & D_{34}^{[1]} \\
D_{41}^{[1]} & D_{42}^{[1]} & D_{43}^{[1]} & D_{44}^{[1]}
\end{array}\right]
$$

while the vectors $\bar{Y}_{m+1}, \bar{Y}_{m}^{[1]}, \bar{Y}_{m}^{[2]}, \bar{Y}_{m}^{[3]}, \bar{F}_{m}^{[0]}, \bar{F}_{m}^{[1]}, \bar{G}_{m}^{[0]}$, and $\bar{G}_{m}^{[1]}$ are defined as follows:

$$
\begin{gathered}
\bar{Y}_{m+1}=\left[\begin{array}{c}
y_{n+r} \\
y_{n+s} \\
y_{n+t} \\
y_{n+1}
\end{array}\right], \\
\bar{Y}_{m}^{[1]}=\left[\begin{array}{c}
y_{n-t} \\
y_{n-s} \\
y_{n-r} \\
y_{n}
\end{array}\right],
\end{gathered}
$$




$$
\begin{gathered}
\bar{Y}_{m}^{[2]}=\left[\begin{array}{c}
y_{n-t}^{\prime} \\
y_{n-s}^{\prime} \\
y_{n-r}^{\prime} \\
y_{n}^{\prime}
\end{array}\right], \\
\bar{Y}_{m}^{[3]}=\left[\begin{array}{l}
y_{n-t}^{\prime \prime} \\
y_{n-s}^{\prime \prime} \\
y_{n-r}^{\prime \prime} \\
y_{n}^{\prime \prime}
\end{array}\right], \\
\bar{Y}_{m}^{[4]}=\left[\begin{array}{c}
y_{n-t}^{\prime \prime \prime} \\
y_{n-s}^{\prime \prime \prime} \\
y_{n-r}^{\prime \prime \prime} \\
y_{n}^{\prime \prime \prime}
\end{array}\right], \\
\bar{F}_{m}^{[0]}=\left[\begin{array}{l}
f_{n-3} \\
f_{n-2} \\
f_{n-1} \\
f_{n}
\end{array}\right], \\
\bar{G}_{m}^{[0]}=\left[\begin{array}{c}
g_{n-3} \\
g_{n-2} \\
g_{n-1} \\
g_{n}
\end{array}\right], \\
\bar{F}_{m}^{[1]}=\left[\begin{array}{l}
g_{n+r} \\
f_{n+s} \\
g_{n+s} \\
g_{n+t} \\
g_{n+1}
\end{array}\right],
\end{gathered}
$$

substituting $q=\lambda h$ in (20) yields

$$
\bar{Y}_{m+1}=M(q) \bar{Y}_{m}^{[1]},
$$

where the amplification matrix $M(q)$ is given by

$$
\begin{gathered}
M(q)=\left(\bar{A}^{[0]}-q^{4} \bar{B}^{[1]}-q^{5} \bar{D}^{[1]}\right)^{-1}\left(\bar{A}^{[1]}+q \bar{A}^{[2]}\right. \\
\left.+q^{2} \bar{A}^{[3]}+q^{3} \bar{A}^{[4]}+q^{4} \bar{B}^{[0]}+q^{5} \bar{D}^{[0]}\right)
\end{gathered}
$$

The analysis shows that the matrix $M(q)$ has eigenvalues $\left\{\eta_{1}, \eta_{2}, \eta_{3}, \eta_{4}\right\}=\left\{0,0,0, \eta_{4}\right\}$, where the dominant eigenvalue

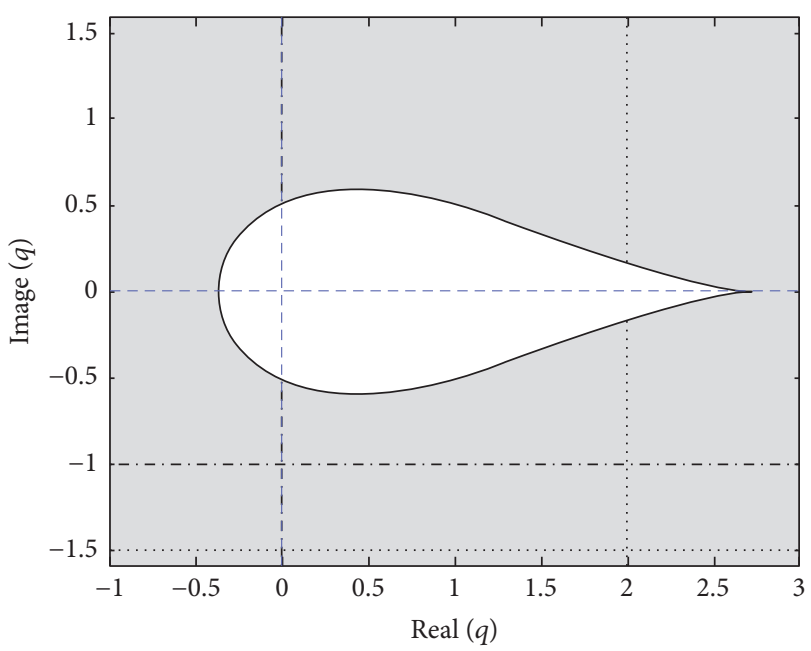

FIGURE 1: Region of absolute stability.

$\eta_{4}$ is a function of $q$ subject to the values $\{r, s, t\}=\{1 / 4$, $1 / 2,3 / 4\}$ given by

$$
\eta_{4}=\frac{\sum_{i=0}^{20} c_{i} q^{i}}{K \sum_{j=0}^{21} c_{j} q^{j}}
$$

where $K=10376293541461622784$ and the values $c_{i}$ and $c_{j}$ are listed in Table 1.

The region of absolute stability is depicted in the dark area in Figure 1.

3.6. Pseudocode of the Developed Method. The following pseudocode illustrates how the developed method is implemented for solving fourth-order initial value problems.

Step 1. The values $y\left(x_{0}\right), y^{\prime}\left(x_{0}\right), y^{\prime \prime}\left(x_{0}\right), y^{\prime \prime \prime}\left(x_{0}\right)$ on the interval $[a, b]$ from the problem are given.

Step 2. Set the values of the hybrid points $\{r, s, t\}$ and step size $h$.

Step 3. For $j=a: h: b$, set $x_{n+j}=x_{n}+j h, j=\{r, s, t, 1\}$.

Step 4. Evaluate the approximate values $y\left(x_{n+j}\right), y^{\prime}\left(x_{n+j}\right)$, $y^{\prime \prime}\left(x_{n+j}\right), y^{\prime \prime \prime}\left(x_{n+j}\right)$ using the direct hybrid block method.

Step 5. Find the solution for the resultant system using the built in function fsolve in Matlab.

Step 6. Calculate the maximum error $=\mid$ Exact solution approximate solution|.

\section{Numerical Examples}

The general three-hybrid one-step hybrid block method (9) with order $p=10$ in this section was employed to solve five problems from the literature. In order to show the performance of the proposed method, the problems are 
TABLE 1

\begin{tabular}{|c|c|c|}
\hline$c$-value & $q^{i}$ coefficients & $q^{j}$ coefficients \\
\hline$c_{0}$ & -19991259433786613699768237287038031044280320 & 0 \\
\hline$c_{1}$ & -19991259433786613699768237287038031044280320 & 192662816967402505371648000 \\
\hline$c_{2}$ & -99956297168933068498841186435190155221401600 & 0 \\
\hline$c_{3}$ & -33318765722977689499613728811730051740467200 & 0 \\
\hline$c_{4}$ & -78083522390790487043756727396404132472422400 & 0 \\
\hline$c_{5}$ & -12270936643443632883203625005043197725900800 & 502432963738164264960000 \\
\hline$c_{6}$ & -994813550529796720162894578620563036569600 & -79502926099016318976000 \\
\hline$c_{7}$ & 59773830578760679822424316692208078028800 & 0 \\
\hline$c_{8}$ & 31534047193242772464338416945297159618560 & 0 \\
\hline$c_{9}$ & 4794949827573668397142419401567455150080 & 133171866570211983360 \\
\hline$c_{10}$ & 359049264395827172053492731789485015040 & -14458011284982988800 \\
\hline$c_{11}$ & -3356754007384253296917217569574748160 & 268452685173657600 \\
\hline$c_{12}$ & -4527291309580589178991255608586403840 & 0 \\
\hline$c_{13}$ & -632549152682975031987854772642562048 & 5775969118252032 \\
\hline$c_{14}$ & -50983394698341254755895386446446592 & -659482112163840 \\
\hline$c_{15}$ & -2347009723724983385107341899462400 & 26223671235440 \\
\hline$c_{16}$ & 3581359970953878842628978864128 & -529590387200 \\
\hline$c_{17}$ & 8897056249188627790299370721984 & 67083520896 \\
\hline$c_{18}$ & 741826115832694210392858425664 & -8638140672 \\
\hline$c_{19}$ & 30585824514148316274163930311 & 506275974 \\
\hline$c_{20}$ & 582915589719914249658541800 & -15909210 \\
\hline$c_{21}$ & 0 & 189567 \\
\hline
\end{tabular}

solved with different values of step size $h$ and hybrid points $\{r, s, t\}$.

Problem 1 (linear fourth-order problem).

$$
\begin{aligned}
& y^{(i v)}=y^{\prime \prime \prime}+y^{\prime \prime}+y^{\prime}+2 y, \\
& y(0)=0, x \leq 2, \\
& y^{\prime}(0)=0, \\
& y^{\prime \prime}(0)=0, \\
& y^{\prime \prime \prime}(0)=30 .
\end{aligned}
$$

Exact Solution. $y=2 e^{2 x}-5 e^{-x}+3 \cos (x)-9 \sin (x)$.

Source. See [15].

Problem 2 (nonlinear fourth-order problem).

$$
\begin{aligned}
& y^{(i v)}=\left(y^{\prime}\right)^{2}-y y^{\prime \prime \prime}-4 x^{2}+e^{x}\left(1+x^{2}-4 x\right), \\
& y(0)=1, \\
& y^{\prime}(0)=1,
\end{aligned}
$$

$$
\begin{aligned}
& y^{\prime \prime}(0)=3, \\
& y^{\prime \prime \prime}(0)=1 .
\end{aligned}
$$

Exact Solution. $y=x^{2}+e^{x}$ with $h=1 / 10$.

Source. See [15].

Problem 3 (application to problem from ship dynamics). In this example, the derived method is implemented to solve a physical problem from ship dynamics. As stated by [3], when a sinusoidal wave of frequency passes along a ship or offshore structure, the resultant fluid actions vary with time. In a particular case study by [3], the fourth-order problem is defined as

$$
y^{(i v)}+3 y^{\prime \prime}+y(2+\epsilon \cos (\omega(x)))=0
$$

$$
x>0 \text {, }
$$

$$
\begin{aligned}
& y(0)=1, \\
& y^{\prime}(0)=y^{\prime \prime}(\rho)=y^{\prime \prime \prime}(0)=0 .
\end{aligned}
$$

Exact Solution. $y(x)=2 \cos (x)+\cos (\sqrt{2} x)$.

Problem 4 (system of linear fourth-order IVPs).

$$
\begin{aligned}
y_{1}^{(i v)} & =y_{1}+y_{4}-e^{2 x}, \\
y(0) & =1, \\
y^{\prime}(0) & =1,
\end{aligned}
$$




$$
\begin{gathered}
y^{\prime \prime}(0)=1, \\
y^{\prime \prime \prime}(0)=1,
\end{gathered}
$$$$
0 \leq x \leq 2,
$$

$$
y_{2}^{(i v)}=y_{2}+y_{3}-x e^{x}
$$$$
y(0)=1,
$$$$
y^{\prime}(0)=-1,
$$$$
y^{\prime \prime}(0)=1,
$$$$
y^{\prime \prime \prime}(0)=-1 \text {, }
$$

$$
0 \leq x \leq 2
$$

$$
\begin{aligned}
y_{3}^{(i v)} & =4 y_{1}+y_{3}, \\
y(0) & =0, \\
y^{\prime}(0) & =1, \\
y^{\prime \prime}(0) & =2, \\
y^{\prime \prime \prime}(0) & =3,
\end{aligned}
$$

$$
0 \leq x \leq 2,
$$

$$
\begin{aligned}
y_{4}^{(i v)} & =y_{1}+y_{2}+16 y_{4}-e^{x}-e^{-x}, \\
y(0) & =1, \\
y^{\prime}(0) & =2 \\
y^{\prime \prime}(0) & =4 \\
y^{\prime \prime \prime}(0) & =8
\end{aligned}
$$

$$
0 \leq x \leq 2,
$$

Exact Solution. $y_{1}=e^{x}, y_{2}=e^{-x}, y_{3}=x e^{x}$, and $y_{4}=e^{2 x}$ with $h=1 / 5$.

Problem 5 (system of nonlinear fourth-order IVPs).

$$
\begin{aligned}
y_{1}^{(i v)} & =y_{1}+y_{2}-\cos (x), \\
y(0) & =0, \\
y^{\prime}(0) & =1, \\
y^{\prime \prime}(0) & =0, \\
y^{\prime \prime \prime}(0) & =-1,
\end{aligned}
$$

$$
\begin{aligned}
& y_{2}^{(i v)}=y_{1}+y_{2}-\sin (x), \\
& y(0)=1,
\end{aligned}
$$

$$
y^{\prime}(0)=0,
$$$$
y^{\prime \prime}(0)=-1 \text {, }
$$$$
y^{\prime \prime \prime}(0)=0 \text {, }
$$

$$
0 \leq x \leq \pi
$$

$$
y_{3}^{(i v)}=32 y_{1} y_{2}
$$$$
y(0)=0,
$$

$$
y^{\prime}(0)=2 \text {, }
$$

$$
y^{\prime \prime}(0)=0 \text {, }
$$$$
y^{\prime \prime \prime}(0)=-8 \text {, }
$$

$$
y_{4}^{(i v)}=16\left(y_{2}^{2}-y_{1}^{2}\right)
$$$$
y(0)=1,
$$

$$
y^{\prime}(0)=0 \text {, }
$$$$
y^{\prime \prime}(0)=-2 \text {, }
$$$$
y^{\prime \prime \prime}(0)=0 \text {, }
$$

$$
0 \leq x \leq \pi
$$

Exact Solution. $y_{1}=\sin (x), y_{2}=\cos (x), y_{3}=\sin (2 x)$, and $y_{4}=x^{2}+\cos (2 x)$, with $h=\pi / 5$.

\section{Conclusion}

A hybrid one-step block method of order $p=10$ with generalized three-off-step points has been derived successfully. The developed method was employed to solve general fourth-order IVPs of ODEs directly. The numerical analysis performed shows that the developed method is consistent and zero stable which leads to the convergence of the method. The numerical results were then compared with the results obtained by the existing methods in terms of error. The new method is found to have better performance over the other methods (refer to Tables 2-12).

\section{Conflicts of Interest}

The authors declare that there are no conflicts of interest regarding the publication of this paper. 
TABLE 2: Comparison of the derived method with $[15,16]$ and Adams Bashforth-Adams Moulton method.

\begin{tabular}{|c|c|c|}
\hline Step size & Method employed & Absolute error at $x=2$ \\
\hline \multirow{4}{*}{0.1} & Derived method & $1.34 E(-11)$ \\
\hline & BHCM4 & $1.74 E(-8)$ \\
\hline & Jator & $1.26 E(-4)$ \\
\hline & Adams & $2.11 E(-3)$ \\
\hline \multirow{4}{*}{0.05} & Derived method & $1.95 E(-11)$ \\
\hline & BHCM4 & $8.45 E(-11)$ \\
\hline & Jator & $1.91 E(-6)$ \\
\hline & Adams & $5.37 E(-4)$ \\
\hline
\end{tabular}

TABLE 3: Comparison of the derived method with $[15,17]$ and Adams Bashforth-Adams Moulton method.

\begin{tabular}{|c|c|c|}
\hline Step size & Method employed & Absolute error at $x=1$ \\
\hline \multirow{4}{*}{0.2} & Derived method & $2.22 E(-15)$ \\
\hline & BHCM4 & $2.38 E(-12)$ \\
\hline & Adams & $5.01 E(-7)$ \\
\hline & Awoyemi & $5.84 E(-4)$ \\
\hline \multirow{4}{*}{0.1} & Derived method & $1.77 E(-15)$ \\
\hline & BHCM4 & $1.95 E(-14)$ \\
\hline & Adams & $2.44 E(-6)$ \\
\hline & Awoyemi & $9.26 E(-5)$ \\
\hline
\end{tabular}

TABLE 4: Comparison of the proposed method with [15] for the case $\epsilon=0$.

\begin{tabular}{|c|c|c|}
\hline Step size & Method employed & Absolute error at $x=15$ \\
\hline \multirow{4}{*}{0.25} & Derived method & $1.1 E(-16)$ \\
\hline & BHCM4 & $5.2 E(-7)$ \\
\hline & Twizell & $1.9 E(-4)$ \\
\hline & Adams & $4.9 E(-3)$ \\
\hline \multirow{4}{*}{0.1} & Derived method & $1.1 E(-14)$ \\
\hline & BHCM4 & $2.8 E(-10)$ \\
\hline & Cortell & $3.7 E(-5)$ \\
\hline & Adams & $8.4 E(-5)$ \\
\hline
\end{tabular}

TABLE 5: Exact and approximate solutions for solving $y_{1}$ using the developed method.

\begin{tabular}{lclc}
\hline$X$-value & Exact solution & Approximate solution & Error in solving $y_{1}$ \\
\hline 0.20 & 1.221402758160169900 & 1.221402758160169900 & $0.000000 E(+00)$ \\
0.40 & 1.491824697641270300 & 1.491824697641270300 & $0.000000 E(+00)$ \\
0.60 & 1.822118800390509100 & 1.822118800390508900 & $2.220446 E(-16)$ \\
0.80 & 2.225540928492467900 & 2.225540928492467400 & $4.440892 E(-16)$ \\
1.00 & 2.718281828459045500 & 2.718281828459045100 & $4.440892 E(-16)$ \\
1.20 & 3.320116922736547200 & 3.320116922736547700 & $4.440892 E(-16)$ \\
1.40 & 4.055199966844674500 & 4.055199966844674500 & $0.000000 E(+00)$ \\
1.60 & 4.953032424395114000 & 4.953032424395114900 & $8.881784 E(-16)$ \\
1.80 & 6.049647464412944800 & 6.049647464412945700 & $8.881784 E(-16)$ \\
2.00 & 7.389056098930648600 & 7.389056098930650400 & $1.776357 E(-15)$ \\
\hline
\end{tabular}


TABLE 6: Exact and approximate solutions for solving $y_{2}$ using the developed method.

\begin{tabular}{lccc}
\hline$X$-value & Exact solution & Approximate solution & Error in solving $y_{2}$ \\
\hline 0.20 & 0.818730753077981820 & 0.818730753077981820 & $0.000000 E(+00)$ \\
0.40 & 0.670320046035639330 & 0.670320046035639330 & $0.000000 E(+00)$ \\
0.60 & 0.548811636094026390 & 0.548811636094026390 & $0.000000 E(+00)$ \\
0.80 & 0.449328964117221560 & 0.449328964117221620 & $5.551115 E(-17)$ \\
1.00 & 0.367879441171442330 & 0.367879441171442330 & $0.000000 E(+00)$ \\
1.20 & 0.301194211912202080 & 0.301194211912202080 & $0.000000 E(+00)$ \\
1.40 & 0.246596963941606490 & 0.246596963941606460 & $2.775558 E(-17)$ \\
1.60 & 0.201896517994655440 & 0.201896517994655380 & $5.551115 E(-17)$ \\
1.80 & 0.165298888221586560 & 0.165298888221586500 & $5.551115 E(-17)$ \\
2.00 & 0.135335283236612730 & 0.135335283236612650 & $8.326673 E(-17)$ \\
\hline
\end{tabular}

TABLE 7: Exact and approximate solutions for solving $y_{3}$ using the developed method.

\begin{tabular}{lccc}
\hline$X$-value & Exact solution & Approximate solution & Error in solving $y_{3}$ \\
\hline 0.20 & 0.244280551632033990 & 0.244280551632033970 & $2.775558 E(-17)$ \\
0.40 & 0.596729879056508210 & 0.596729879056508090 & $1.110223 E(-16)$ \\
0.60 & 1.093271280234305600 & 1.093271280234305400 & $2.220446 E(-16)$ \\
0.80 & 1.780432742793974300 & 1.780432742793974100 & $2.220446 E(-16)$ \\
1.00 & 2.718281828459045500 & 2.718281828459045100 & $4.440892 E(-16)$ \\
1.20 & 3.984140307283856600 & 3.984140307283857000 & $4.440892 E(-16)$ \\
1.40 & 5.677279953582544000 & 5.677279953582544000 & $0.000000 E(+00)$ \\
1.60 & 7.924851879032181600 & 7.924851879032183400 & $1.776357 E(-15)$ \\
1.80 & 10.889365435943299000 & 10.889365435943303000 & $3.552714 E(-15)$ \\
2.00 & 14.778112197861295000 & 14.778112197861301000 & $5.329071 E(-15)$ \\
\hline
\end{tabular}

TABLE 8: Exact and approximate solutions for solving $y_{4}$ using the developed method.

\begin{tabular}{lccc}
\hline$X$-value & Exact solution & Approximate solution & Error in solving $y_{4}$ \\
\hline 0.20 & 1.491824697641270300 & 1.491824697641270300 & $0.000000 E(+00)$ \\
0.40 & 2.225540928492467900 & 2.225540928492467400 & $4.440892 E(-16)$ \\
0.60 & 3.320116922736548100 & 3.320116922736547700 & $4.440892 E(-16)$ \\
0.80 & 4.953032424395114900 & 4.953032424395114900 & $0.000000 E(+00)$ \\
1.00 & 7.389056098930650400 & 7.389056098930650400 & $0.000000 E(+00)$ \\
1.20 & 11.023176380641601000 & 11.023176380641601000 & $0.000000 E(+00)$ \\
1.40 & 16.444646771097048000 & 16.444646771097048000 & $0.000000 E(+00)$ \\
1.60 & 24.532530197109342000 & 24.532530197109349000 & $7.105427 E(-15)$ \\
1.80 & 36.598234443677974000 & 36.598234443677988000 & $1.421085 E(-14)$ \\
2.00 & 54.598150033144215000 & 54.598150033144236000 & $2.131628 E(-14)$ \\
\hline
\end{tabular}

TABLE 9: Exact and approximate solutions for solving $y_{1}$ using the developed method.

\begin{tabular}{lccc}
\hline$X$-value & Exact solution & Approximate solution & Error in solving $y_{1}$ \\
\hline 0.6283185 & 0.587785252292473140 & 0.587785252292475800 & $2.664535 E(-15)$ \\
1.2566371 & 0.951056516295153530 & 0.951056516295303300 & $1.497691 E(-13)$ \\
1.8849556 & 0.951056516295153640 & 0.951056516296290070 & $1.136424 E(-12)$ \\
2.5132741 & 0.587785252292473250 & 0.587785252297075340 & $4.602096 E(-12)$ \\
3.1415927 & 0.000000000000000122 & 0.000000000013537650 & $1.353753 E(-11)$ \\
\hline
\end{tabular}


TABLE 10: Exact and approximate solutions for solving $y_{2}$ using the developed method.

\begin{tabular}{lccc}
\hline$X$-value & Exact solution & Approximate solution & Error in solving $y_{2}$ \\
\hline 0.6283185 & 0.809016994374947450 & 0.8090169943749555000 & $7.549517 E(-15)$ \\
1.2566371 & 0.309016994374947450 & 0.309016994375326810 & $3.793632 E(-13)$ \\
1.8849556 & -0.309016994374947340 & -0.309016994372823590 & $2.123746 E(-12)$ \\
2.5132741 & -0.809016994374947340 & -0.809016994368452200 & $6.495138 E(-12)$ \\
3.1415927 & -1.000000000000000000 & -0.999999999984998780 & $1.500122 E(-11)$ \\
\hline
\end{tabular}

TABLE 11: Exact and approximate solutions for solving $y_{3}$ using the developed method.

\begin{tabular}{lccc}
\hline$X$-value & Exact solution & Approximate solution & Error in solving $y_{3}$ \\
\hline 0.6283185 & 0.951056516295153530 & 0.951056516302223540 & $7.070011 E(-12)$ \\
1.2566371 & 0.587785252292473250 & 0.587785252378536070 & $8.606282 E(-11)$ \\
1.8849556 & -0.587785252292473030 & -0.587785251895384220 & $3.970888 E(-10)$ \\
2.5132741 & -0.951056516295153640 & -0.951056515173170910 & $1.121983 E(-09)$ \\
3.1415927 & -0.000000000000000245 & 0.000000002364014064 & $2.364014 E(-09)$ \\
\hline
\end{tabular}

TABLE 12: Exact and approximate solutions for solving $y_{4}$ using the developed method.

\begin{tabular}{lccc}
\hline$X$-value & Exact solution & Approximate solution & Error in solving $y_{4}$ \\
\hline 0.6283185 & 0.703801170418521750 & 0.703801170427732160 & $9.210410 E(-12)$ \\
1.2566371 & 0.770119709799349850 & 0.770119709892383430 & $9.303358 E(-11)$ \\
1.8849556 & 2.744040590017221300 & 2.744040590323378700 & $3.061573 E(-10)$ \\
2.5132741 & 6.625563811072136200 & 6.625563811678808300 & $6.066720 E(-10)$ \\
3.1415927 & 10.869604401089358000 & 10.869604401982164000 & $8.928058 E(-10)$ \\
\hline
\end{tabular}

\section{Acknowledgments}

The authors thank Universiti Utara Malaysia for the financial support of the publication of this article.

\section{References}

[1] A. Malek and R. Shekari Beidokhti, "Numerical solution for high order differential equations using a hybrid neural network-optimization method," Applied Mathematics and Computation, vol. 183, no. 1, pp. 260-271, 2006.

[2] A. Boutayeb and A. Chetouani, "A mini-review of numerical methods for high-order problems," International Journal of Computer Mathematics, vol. 84, no. 4, pp. 563-579, 2007.

[3] X. J. Wu, Y. Wang, and W. G. Price, "Multiple resonances, responses, and parametric instabilities in offshore structures," Journal of Ship Research, vol. 32, no. 4, pp. 285-296, 1988.

[4] W. E. Milne, Numerical Solution of Differential Equations, John Wiley \& Sons, New York, NY, USA, 1953.

[5] J. B. Rosser, "A runge-kutta for all seasons," SIAM Review, vol. 9, no. 3, pp. 417-452, 1967.

[6] W. B. Gragg and H. J. Stetter, "Generalized multistep predictorcorrector methods," Journal of the ACM, vol. 11, no. 2, pp. 188209, 1964

[7] J. D. Lambert, Computational Methods in Ordinary Differential Equations, John Wiley \& Sons, New York, NY, USA, 1973.

[8] G. Dahlquist, "Convergence and stability in the numerical integration of ordinary differential equations," Mathematica Scandinavica, vol. 4, pp. 33-53, 1956.
[9] W. H. Enright, "Second derivative multistep methods for stiff ordinary differential equations," SIAM Journal on Numerical Analysis, vol. 11, no. 2, pp. 321-331, 1974.

[10] F. F. Ngwane and S. N. Jator, "Block hybrid-second derivative method for stiff systems," International Journal of Pure and Applied Mathematics, vol. 80, no. 4, pp. 543-559, 2012.

[11] R. K. Sahi, S. N. Jator, and N. A. Khan, "A simpson's-type second derivative method for stiff systems," International Journal of Pure and Applied Mathematics, vol. 81, no. 4, pp. 619-633, 2012.

[12] R. K. Sahi, S. N. Jator, and N. A. Khan, "Continuous fourth derivative method for third order boundary value problems," International Journal of Pure and Applied Mathematics, vol. 85, no. 5, pp. 907-923, 2013.

[13] S. O. Fatunla, Numerical Methods for Fatunla Initial Value Problems in Ordinary Differential Equations, Academic Press, New York, NY, USA, 1988.

[14] P. Henrici, Discrete Variable Methods in ODEs, John Wiley \& Sons, New York, NY, USA, 1962.

[15] L. K. Yap and F. Ismail, "Block hybrid collocation method with application to fourth order differential equations," Mathematical Problems in Engineering, vol. 2015, Article ID 561489, 6 pages, 2015.

[16] S. N. Jator, "Numerical integrators for fourth order initial and boundary value problems," International Journal of Pure and Applied Mathematics, vol. 47, no. 4, pp. 563-576, 2008.

[17] D. O. Awoyemi, "Algorithmic collocation approach for direct solution of fourth-order initial-value problems of ordinary differential equations," International Journal of Computer Mathematics, vol. 82, no. 3, pp. 321-329, 2005. 


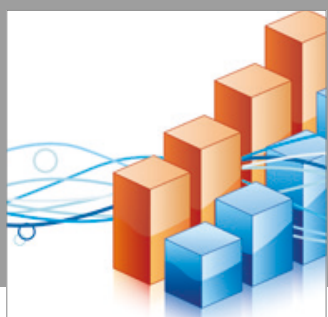

Advances in

Operations Research

vatersals

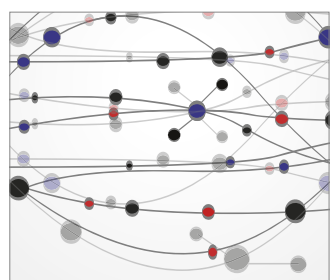

\section{The Scientific} World Journal
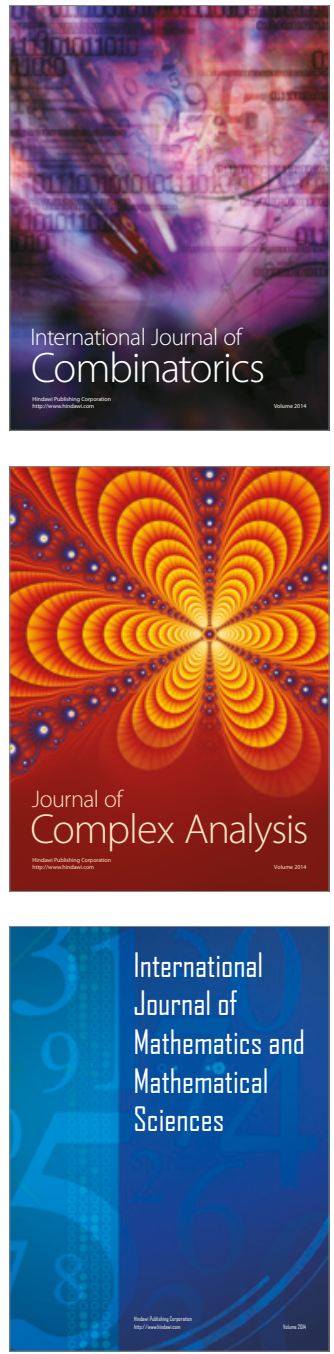
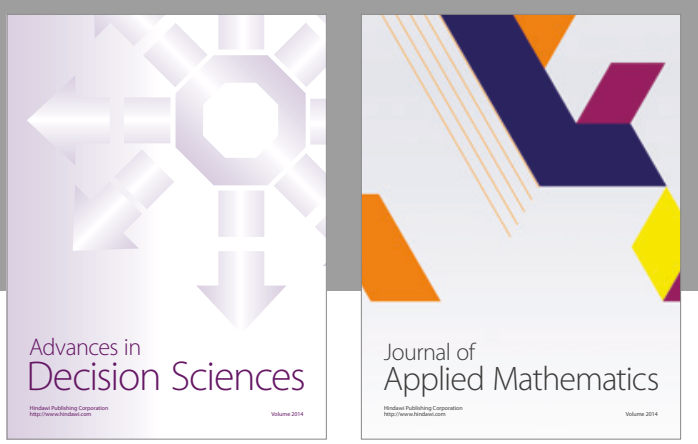

Algebra

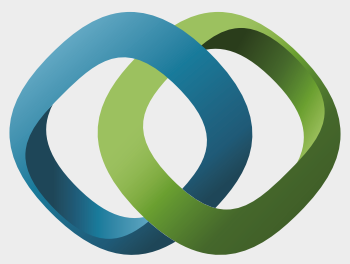

\section{Hindawi}

Submit your manuscripts at

https://www.hindawi.com
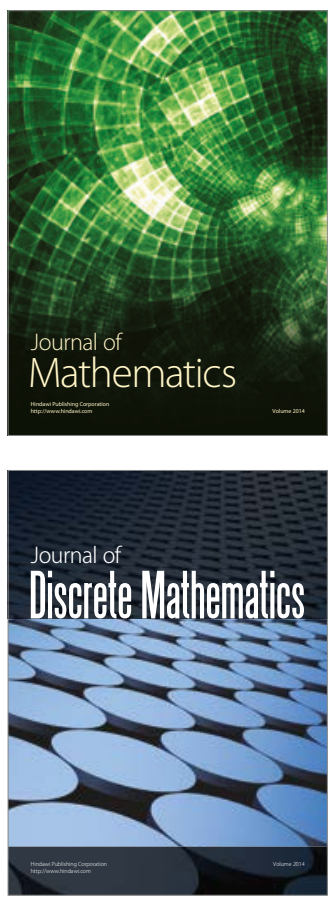

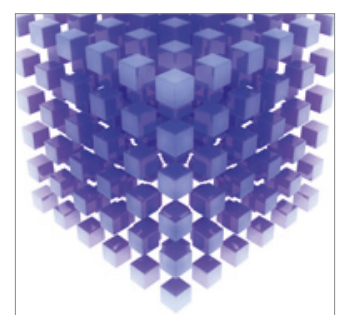

Mathematical Problems in Engineering
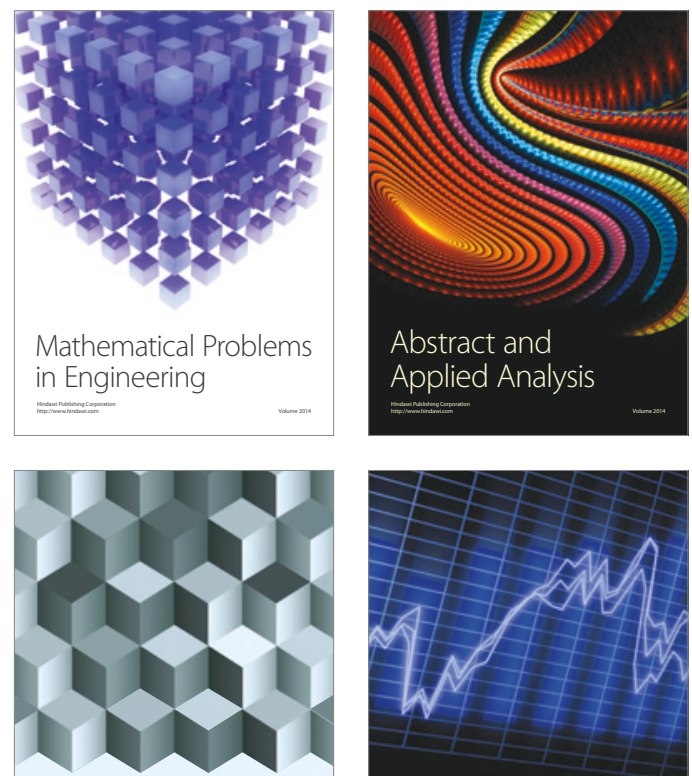

Journal of

Function Spaces

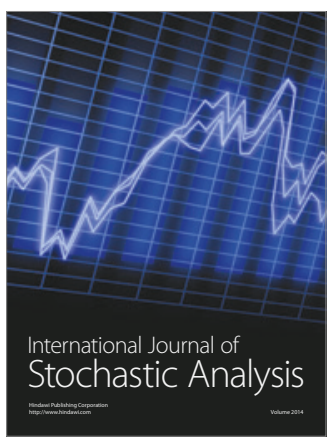

Probability and Statistics
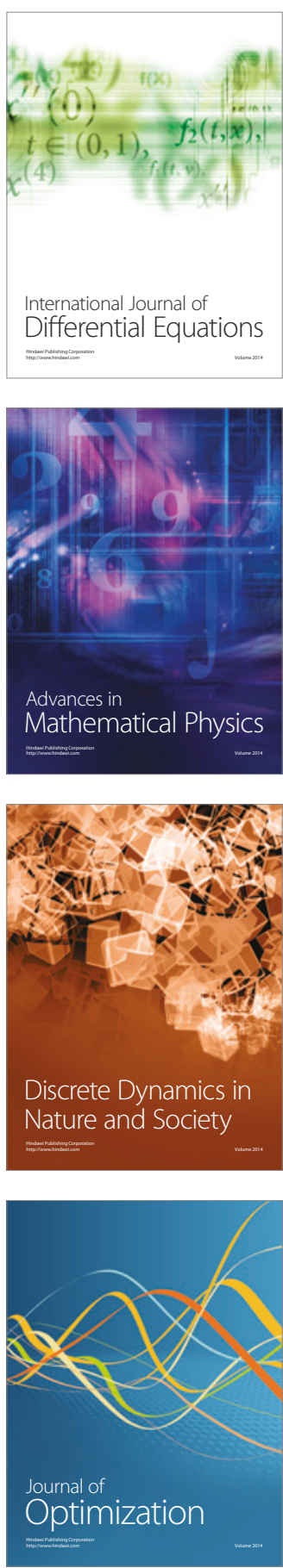